and superb micrographs. Such a beautifully crafted book is rarely seen in science. Given its large format $(24 \mathrm{~cm} \times 34 \mathrm{~cm})$ and its opulent contents, it can be valued as much as a work of visual art as an informative work of biological science. But I wish to emphasize that this is a rigorous scientific monograph. The contents are well referenced to the scientific literature (up to the mid-1990s), and the writing is clear and crisp.

It provides a wide audience with an astoundingly beautiful and admirably accurate account of how a worker honeybee smells and sees, tastes and touches, feeds and breathes, flies and stings, secretes wax and releases pheromones. Goodman has given all who are interested in the behaviour and physiology of bees an amazing gift.

Thomas D. Seeley is in the Department of

Neurobiology and Behavior, Cornell University, Ithaca, New York 14853, USA.

\section{More on bees}

Bumblebees: Behaviour and Ecology by Dave Goulson

Oxford University Press, $£ 55$ ( $h b k$ ), $£ 27.50$ (pbk)

$\bullet \bullet \bullet \bullet \bullet \bullet \bullet \bullet \bullet \bullet \bullet \bullet \bullet \bullet \bullet \bullet \bullet \bullet \bullet \bullet$

\section{Universal support}

\section{Echo of the Big Bang}

by Michael Lemonick

Princeton University Press: 2003. 232 pp.

$\$ 24.95, £ 17.95$

\section{Sean Carroll}

The first pages of Michael Lemonick's new book grab the attention. Princeton University astrophysicist David Spergel is fretting over the implications of a new discovery, made from observations of the cosmic microwave background (CMB), which according to Lemonick implies that "much of the work in cosmology over the past two decades has been based on a faulty theoretical foundation". The observations are from NASA's Wilkinson Microwave Anisotropy Probe (WMAP), a satellite that measures, with unprecedented precision, temperature fluctuations in the relic radiation from the Big Bang.

It is a gripping vignette, but puzzling: as we now know, the WMAP results didn't include any major surprises. If anything, WMAP provided a convincing capstone to a series of impressive advances in cosmology, demonstrating that our consensus picture of the Universe is fairly accurate. This picture can be hard to swallow, as it describes a Universe of which only $4 \%$ is made up of ordinary matter; the rest is a mixture of cold dark matter and an exotic'dark energy', which remains nearly constant throughout space and time. When a purported consensus leans so heavily on the invocation of unseen substances, it is important to verify it from every

\title{
Vesalius's vessels
}

When Andreas Vesalius published his De Humani Corporis Fabrica Libri Septem (On the

Fabric of the Human Body) in 1543, its accurate depiction of the human body from close observation of dissected bodies overturned centuries of medical dogma and led the way for the modern study of anatomy. William Richardson and John Carman have been translating this treatise; the third volume, containing Books III and IV from the original work, focusing on the veins, arteries and nerves, has now been published (Jeremy Norman, \$250). Although the accuracy of Vesalius' work on these soft tissues has not stood the test of time as well as the earlier skeletal work, the illustrations, like that of the portal vein shown here, are stunning. The vessels appear as if fixed in space, with the other internal organs simply invisible.

Mary Purton

www.historyofscience.com

angle; the WMAP results were justly hailed as an impressive example of such a verification.

Of course, the WMAP team did make fresh discoveries, as well as confirming existing views. The satellite measured the polarization of the CMB on large angular scales, which can be interpreted as evidence for re-ionization of the Universe by early star formation. In addition, a few tantalizing features of the WMAP data can be interpreted as evidence for something new, but these are hints rather than firm results; nobody has been moved to overthrow the theoretical foundations of cosmology.

The opening chapter does not explain the nature of the unsettling discovery to which it refers; it is a teaser, meant to draw the reader into the rest of the narrative. The mystery is only revealed near the end, where we learn that it refers to the possibility that the data indicate a finite spatial size to the Universe. But we also learn that this possibility is not taken very seriously; Spergel is quoted as saying: "We don't think it means anything." And Lemonick concludes the book by admitting that the WMAP team "wouldn't be saying anything shocking". A reader might feel somewhat cheated at this point.

This episode is emblematic of the frustrating nature of the book. Lemonick was given access to the inner workings of the WMAP team (as an embedded journalist, if you will), a collaboration whose deliberations were closely guarded from the rest of the scientific community. But Lemonick seems to want to tell a slightly different story to the one that actually happened - one in which a heroic band of plucky scientists raced against outside competition and fought valiantly against recalcitrant NASA bureaucracy, succeeding in the end in redrawing our picture of the Universe. In reality, the talented and dedicated WMAP team worked within the context of numerous experiments, taking complementary approaches to understanding cosmology. The result was a triumph of the congratulatory rather than the revolutionary kind: we had it right all along.

The insider's view of how real scientists go about their everyday business is one of this book's strongest points. The human element is well illuminated, as in the account of the excitement and pressure accompanying the satellite launch itself. Indeed, human concerns are perhaps emphasized too much; the struggle over whether satellite components were to be assembled on Princeton's campus or at a NASA centre is likely to be less fascinating to the general reader than it was to the participants at the time.

A more important complaint is that the book takes too many shortcuts in explaining the science behind the CMB observations. It is hard to understand, for example, why the most visually striking product of the WMAP observations - a high-resolution map of the CMB fluctuations - is not included. For some reason, a simulation of the map is reproduced, but not the map itself.

WMAP (named after David Wilkinson, a team member and $\mathrm{CMB}$ pioneer who died in 2002) marks the end of a decade of upheaval and triumph in cosmology, which began with the original discovery in 1992 of CMB fluctuations by COBE, another NASA satellite. This book describes a small but important part of the achievements of the past 11 years. A comprehensive view of this most exciting time in the history of cosmology is a worthy story that remains to be told. Sean Carroll is at the Enrico Fermi Institute,

University of Chicago, 5640 South Ellis Avenue, Chicago, Illinois 60637, USA. 